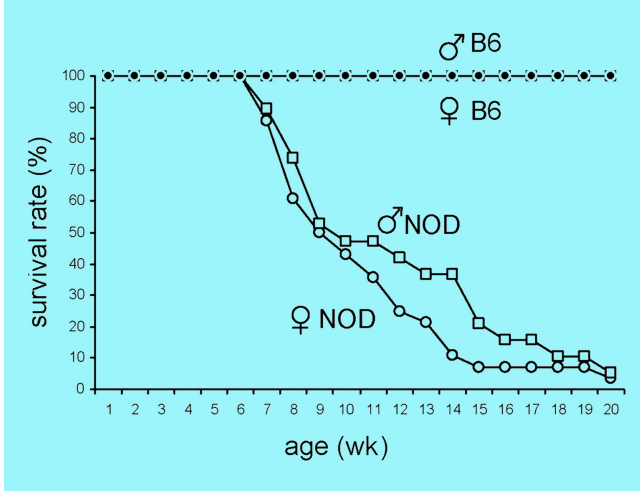

Aire deficiency causes lethal autoimmune disease in NOD mice, but only mild disease in C57BL/6 (B6) mice.

\section{Modifying Aire}

Genetic background can dictate the target and severity of an autoimmune attack that results from a single genetic mutation, according to Jiang and colleagues on page 805 .

Most autoimmune diseases are caused by multiple genetic defects that conspire to break down the immune system's tolerance to selftissues. One exception is autoimmunity caused by mutations in Aire, the transcriptional regulator that controls tolerance by driving the expression of tissue-restricted genes in thymic epithelial cells. Thymic expression of these genes causes self-reactive T cells to be either deleted or tolerized. Mice and humans with mutations in Aire develop autoimmune symptoms that vary widely in target organ and disease severity.

In an attempt to explain this variation, Jiang and colleagues back-crossed Aire-deficient mice onto well-characterized mouse strains and showed that genetic background decides their fate. Autoimmune-prone nonobese diabetic (NOD) mice coped poorly without Aire, suffering severe attacks on multiple organs including the pancreas, stomach, and lung. C57BL/6 mice fared better with a mild disease that was focused primarily on the prostate.

Congenic and intercross analyses of the NOD.Aire A $^{-1-}$ and C57BL/6/Aire ${ }^{-1-}$ mice showed that pancreatitis was controlled by the same MHC haplotype $\left(\mathrm{H}-2^{97}\right)$ that predisposes NOD mice to diabetes, whereas gastritis was controlled by unknown genes on chromosome 3 . Further studies are required to determine how these modifier genes influence the disease manifestations caused by the absence of Aire. JEM

\section{Blocking acid, boosting T cells}

Intracellular antacids boost cytotoxic $\mathrm{T}$ cell responses, according to a study on page 817. Accapezzato and colleagues show that an inhibitor that prevents endosome acidification enhances presentation of antigens to $\mathrm{CD}^{+} \mathrm{T}$ cells and boosts vaccine responses.

Many vaccines are made of soluble proteins derived from viruses or bacteria. But these vaccines tend to be poor stimulators of $\mathrm{CD}^{+} \mathrm{T}$ cells, as soluble antigens are primarily degraded in acidified endosomes; the resulting peptides are loaded onto MHC class II molecules and presented to CD4 ${ }^{+}$ helper T cells. But in certain cell types, exogenous antigen can escape from endosomes and enter the cytosol. From there, the antigen can infiltrate the class I MHC processing pathway-which is normally reserved for internally synthesized proteins - and be cross-presented to $\mathrm{CD}^{+}{ }^{+} \mathrm{T}$ cells.

Accapezzato and colleagues now show that cross-presentation is enhanced when antigen-pulsed dendritic cells are treated with chloroquine, a widely used antimalarial drug that prevents endosome acidification. The enhancement was due to both decreased antigen degradation and increased escape of antigen into the cytosol. The latter finding is consistent with a recent study showing that chloroquine can increase membrane permeability.

A single dose of oral chloroquine given to recipients of hepatitis $\mathrm{B}$ virus booster shots resulted in detectable $\mathrm{CD}^{+} \mathrm{T}$ cell responses in
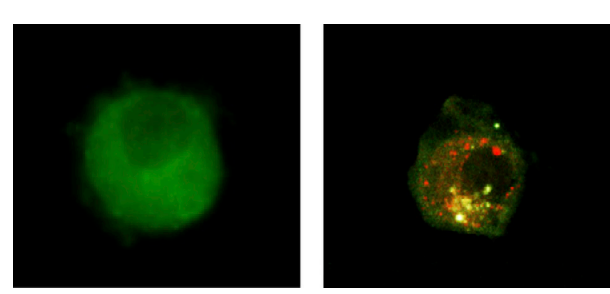

Chloroquine gets soluble antigens out of endosomes (right) and into the cytoplasm (left). nearly $70 \%$ of the recipients, compared with no responses in those who received the shot alone. This readily available drug might thus be a useful $T$ cell-boosting supplement to soluble antigen vaccines. JEM

\section{Evolution of class switching}

Antibody class switching emerged in evolutionary history when bony fish grew legs and became amphibians. On page 733, Barreto and colleagues show that the switch-inducing enzyme AID (activation-induced cytidine deaminase) is in working order in bony fish, suggesting that the evolution of the switch reaction had to wait for the acquisition of some other component.

Bony fish produce antibodies but lack class switch recombination, a process by which the constant region of the antibody heavy chain is swapped for that of a different isotype. This process is missing in fish despite the expression of AID and the presence of antibody somatic hypermutation, a process also initiated by AID.

This group recently showed that $\mathrm{COOH}$-terminal mutations in AID robbed the enzyme of its class-switching function without affecting hypermutation. The zebrafish AID gene contains $\mathrm{COOH}$-terminal mutations, suggesting that fish AID might be unable to recruit essential cofactors or to bind appropriately to the heavy chain locus. But Barreto et al. now show that AID from zebrafish restored normal class switching in AID-deficient mouse B cells, indicating that the functional domains of AID that are required for class switching were all intact.

Thus it appears that the rate-limiting step for class switching was not the evolution of a switch-capable AID protein, but rather the evolution of appropriate DNA switch regions, which are indeed missing from fish heavy chain locus. JEM 


\section{CD23 bridges the gap}

The structure of the low-affinity human immunoglobulin $E(\lg E)$ receptor $C D 23$, revealed on page 751, accounts for its provocative behavior. Hibbert and colleagues show that soluble CD23 (sCD23) can simultaneously bind IgE and the complement receptor $C D 21$ on $B$ cells. This binding dexterity helps explain how $C D 23$ and $\lg E$ cooperate to ramp up the synthesis of more $\lg \mathrm{E}$.

The production of $\mathrm{IgE}$-the signature antibody of allergic diseases-is enhanced when sCD23 (which is cleaved from the surface of activated B cells by endogenous or pathogen-derived proteases) binds to CD21 on other B cells. But it was not clear why sCD23 binding to CD21 enhanced the production of IgE but not other antibody isotypes, as CD21 is present on all B cells.

The group used NMR spectroscopy to show that sCD23 can bind to both $\lg E$ and CD21 simultaneously, as their binding sites are nonoverlapping. This dual binding capacity allows SCD23 to link IgE and CD21 on the surface of B cells. How this linkage triggers $\lg E$ production is not yet known, but the process is likely to be similar to one initiated by the complement component C3d. C3d, when covalently linked to antigen, brings together the $B$ cell receptor (immunoglobulin M) and CD21. This assemblage recruits the $C D 21$-associated signaling molecule $C D 19$, which synergizes with the $B$ cell receptor to increase activation and proliferation of the cell.

Once IgE levels are high, a trimeric membrane-bound form of CD23 helps turn off $\lg E$ production, possibly by stabilizing the molecule and preventing its cleavage. The next challenge, according to senior author James McDonnell, will be to study CD23-mediated $\lg$ E regulation in animal models of allergic disease. In the meantime, these data might provide a structural basis for the development of novel inhibitors of allergic disease. JEM

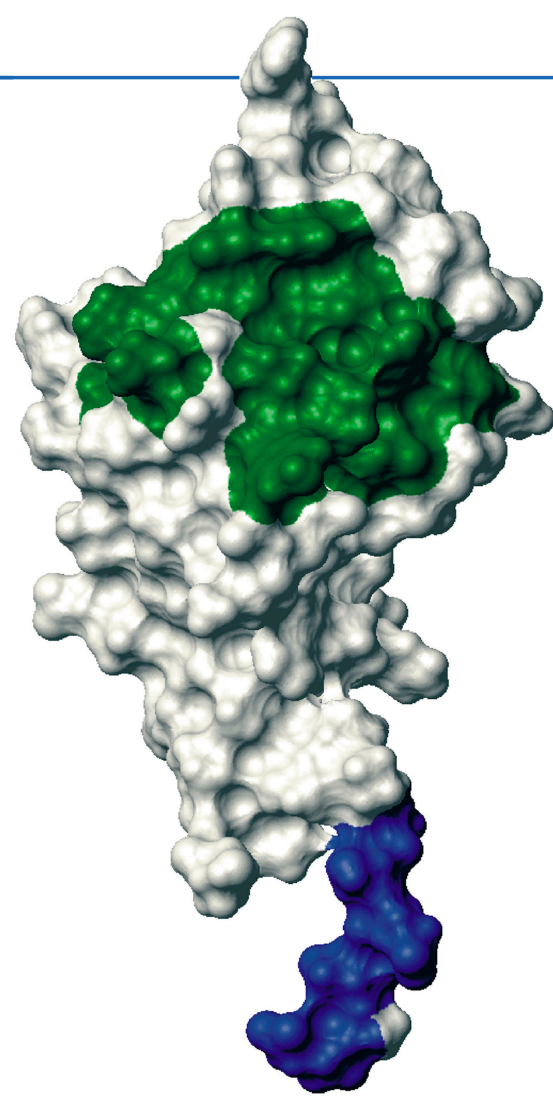

Structure of the CD23 molecule showing the binding sites for IgE (green) and CD21 (blue).

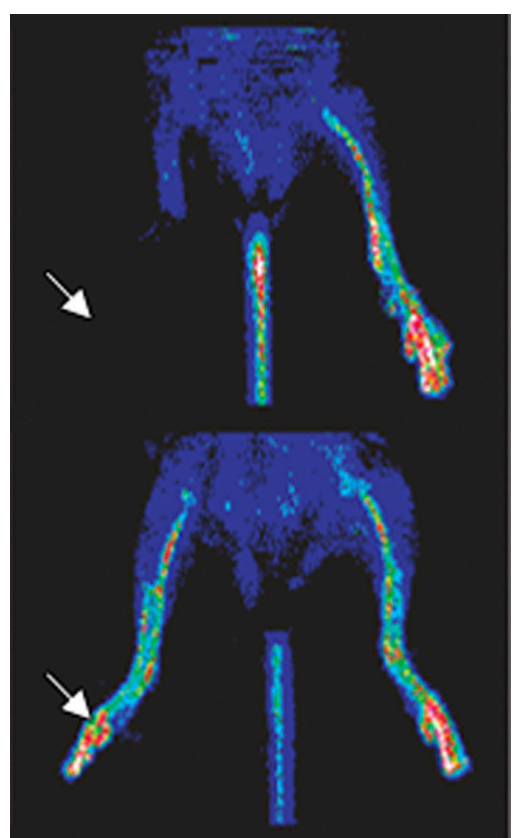

Low-dose irradiation enhances limb regeneration (bottom) unless mast cells are absent (top).

\section{Vessel-building mast cells}

Irradiation instructs mast cells to rebuild blood vessels in oxygen-deprived tissues, according to Heissig and colleagues on page 739 . Low-dose irradiation mobilized adult and precursor mast cells, which traveled to the ischemic tissue and produced the vesselbuilding growth factor VEGF (vascular endothelial growth factor).

Ionizing irradiation has been shown to promote the growth of new blood vessels through the induction of VEGF and inhibition of endothelial cell death. However, the primary source of irradiation-induced VEGF and the precise mechanism that triggers its production were not clear. Mast cells-well-known for their production of histamine in allergic responses- had been found in tissues undergoing angiogenesis. Mast cells are also capable of producing VEGF, but whether these cells play a leading or supporting role in angiogenesis had not been explored.

Heissig et al. now show that mast cells are essential for irradiation-induced vessel growth in a mouse model of ischemic limb injury. Irradiation-induced cellular damage triggers the production of the degradative enzyme matrix metalloprotease-9 (MMP-9) by largely unknown mechanisms. In this model, local irradiation of the damaged limb increased MMP-9 production in the ischemic tissue, which liberated the cytokine Kit ligand (KitL), likely from stromal cells. KitL then attracted VEGFproducing mast cells into the injured tissue.

At the same time, irradiation mobilized mast cell precursors and endothelial cell progenitors from the bone marrow, and some of these cells became incorporated into the growing vessels. Without mast cells, the vessel-building benefits of irradiation were lost. The authors thus speculate that localized irradiation might improve upon current therapies for organ regeneration, which rely on the injection of large numbers of bone marrow-derived stem cells directly into damaged organs. JEM 